
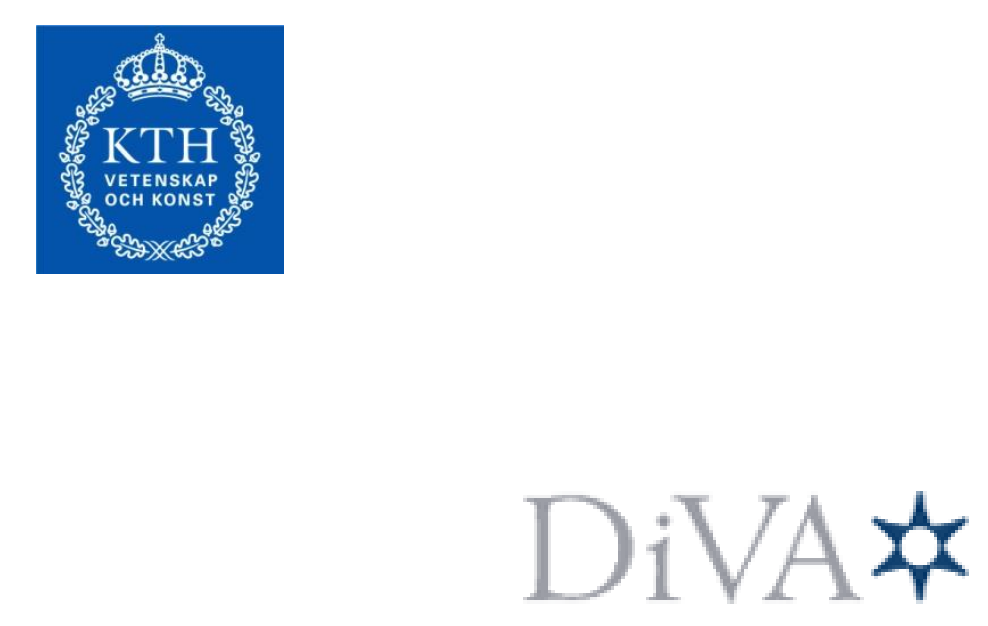

http://kth.diva-portal.org

This is an author produced version of a paper published in IEEE Transactions on Power Delivery, 2011, Vol. 26, No 3, pp. 1372-1380.

This paper has been peer-reviewed but does not include the final publisher proofcorrections or proceedings pagination.

(C) 2012 IEEE. Personal use of this material is permitted. Permission from IEEE must be obtained for all other uses, in any current or future media, including reprinting/republishing this material for advertising or promotional purposes, creating new collective works, for resale or redistribution to servers or lists, or reuse of any copyrighted component of this work in other works.

Citation for the published paper:

Moustafa Chenine and Lars Nordström.

Modeling and Simulation of Wide Area Communication for Centralized PMU-based Applications.

IEEE Transactions on Power Delivery, 2011, Vol. 26, No 3, pp. 1372-1380.

Access to the published version may require subscription.

Published with permission from: IEEE 


\title{
Modeling and Simulation of Wide-Area Communication for Centralized PMU-Based Applications
}

\author{
Moustafa Chenine, Student Member, IEEE, and Lars Nordström, Member, IEEE
}

\begin{abstract}
Phasor-based wide-area monitoring and control (WAMC) systems are becoming a reality with increased research, development, and deployments. Many potential control applications based on these systems are being proposed and researched. These applications are either local applications using data from one or a few phasor measurement units (PMUs) or centralized utilizing data from several PMUs. An aspect of these systems, which is less well researched, is the WAMC system's dependence on high-performance communication systems. This paper presents the results of research performed in order to determine the requirements of transmission system operators on the performance of WAMC systems in general as well as the characteristics of communication delays incurred in centralized systems that utilize multiple PMUs distributed over a large geographic area. This paper presents a summary of requirements from transmission system operators with regards to a specific set of applications and simulations of communication networks with a special focus on centralized applications. The results of the simulations indicate that the configuration of central nodes in centralized WAMC systems needs to be optimized based on the intended WAMC application.
\end{abstract}

Index Terms-Data quality, performance, phasor-measurementunit (PMU)-based applications, wide-area monitoring and control (WAMC) systems power system communication.

\section{INTRODUCTION}

$\mathbf{E}$ LECTRICAL power networks are a part of the critical infrastructure in modern society. Our dependence and demand on electricity has risen sharply while recently this rising demand for electricity has been met with a serious strain in terms of production and expansion of transmission capacity. This is, among other factors, due to increasing environmental policies and costs. Furthermore, the re-regulation of the electrical market and the connection of national grids with neighboring nations have resulted in a more complex and dynamic environment, in which multiple organizations coordinate and cooperate in the operation and control of the power system. As a result, there is

Manuscript received September 16, 2009; revised February 09, 2010, October 04, 2010; accepted December 18, 2010. Date of publication February 24, 2011; date of current version June 24, 2011. This work was supported in part by the Swedish Power industry via ELEKTRA project 36005 and in part by $\mathrm{EKC}^{2}$ - The Swedish Centre of Excellence in Electric Power Engineering. Paper no. TPWRD-00697-2009.

The authors are with the Department of Industrial Information and Control Systems, School of Electrical Engineering, KTH-The Royal Institute of Technology, Stockholm SE-10044, Sweden (e-mail: moustafac@ics.kth.se; larsn@ics.kth.se).

Color versions of one or more of the figures in this paper are available online at http://ieeexplore.ieee.org.

Digital Object Identifier 10.1109/TPWRD.2011.2106805 an international interest and implementation drive, in academia and industry, on the prospects of monitoring and control technology based on PMUs [1], [2]. These systems promise to offer more accurate and timely data on the state of the power system increasing the possibilities to manage the system at a more efficient and responsive level and apply wide-area control and protection schemes.

Generally, most of the international effort [1]-[4] has been on developing monitoring and assessment applications based on PMU measurements, in addition to platforms that would support these applications (e.g., the Gridstat project [5]). Monitoring and assessment applications are known as wide-area monitoring systems (WAMS). These new applications were previously not possible with supervisory-control-and-data-acquistion (SCADA) measurements due to their generally low data sampling rate and lack of exact time synchronization. There has been generally less work on developing protection systems for PMU-based monitoring and assessment applications, and even less so for control applications. This second group of systems which not only monitors the power system states are referred to as wide-area monitoring and control (WAMC) systems.

\section{A. Scope of the Paper}

The scope of this paper is to present a study regarding the architecture of WAMC systems utilizing a large number of PMUs. The hypothesis is that the large amounts of data coupled with strict real-time requirements limits the choices of architectures for implementing centralized WAMC systems. Based on requirements from transmission system operators (TSOs) with regards to WAMC applications, a simulation model of the wide-area communication infrastructure was built. This model was used to simulate the effect of different architectures with regards to dedicated or shared communication links and different data-sorting algorithms. In regards to time delays, previous studies in this field have not considered the impact of certain important architectural components, specifically the phasor data concentrator (PDC), on the overall delay. In this paper, these components and their impacts are taken into considerations.

\section{B. Outline}

The paper is structured as follows; Section II presents an overview of WAMC architectures outlining the main components of these systems. Section III presents a summary of a survey carried out in the Nordic region to determine the overall 


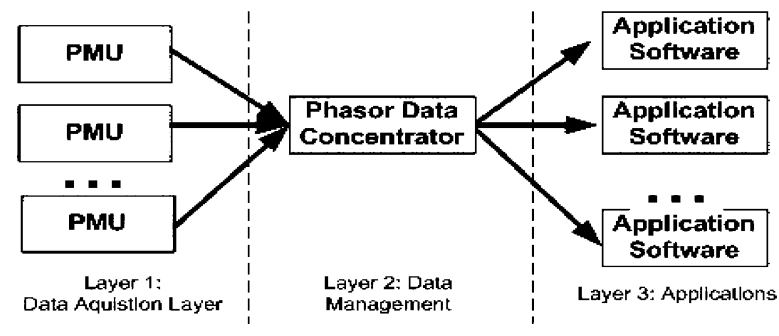

Fig. 1. Layers and components of the WAMC system.

requirements of WAMC systems. Section IV describes the simulation model scenarios built to study wide-area communication delays in WAMC systems. The delays are then applied to study the impact of the PDC on the currency and completeness of the measurement set received at the WAMC application in the control system, this is described in Section V. Finally, the paper is concluded in Section VI.

\section{WAMC ARCHITECTURES}

A complete PMU-based monitoring and control system is a system in which PMU measurements are collected from various locations in the electrical grid in a nation or region. The measurements are then communicated to a central location where they are used by assessment or monitoring applications that would raise alarms or calculate results. The alarms raised and results calculated by these monitoring systems are, in turn, used to provide corrective actions or control on the power grid. This complete PMU-based system is known as a WAMC system. Alternative architectures, such as using a few remote PMU signals in a local system for a specific protection application are also possible, but are outside the scope of this study.

\section{A. WAMC Components}

A WAMC system includes four basic components: 1) a PMU; 2) a PDC; 3) the PMU-based application; and, finally, the communication network [6].

Logically, there are three layers in a WAMC system which, in essence, is very similar to more traditional SCADA systems. Fig. 1 illustrates the logical architecture of WAMC systems. Layer 1 where the WAMC system interfaces with the power system on substation bars and power lines is called the data-acquisition layer, this is where the PMUs are placed. Layer 2 is known as the data-management layer and that is where the PMU measurements are collected and sorted into a single time synchronized dataset. Finally, Layer 3 is the application layer that represents the real-time PMU-based application functions that process the time-synchronized PMU measurements provided by Layer 2.

\section{B. Communication Infrastructure}

The communication infrastructure is an important component in the architecture of a WAMC system. This is because PMU devices are distributed over a wide area, covering various locations within a power system's boundaries. The PMU devices are then connected to a central control center or several control centers over a communication network. The communication

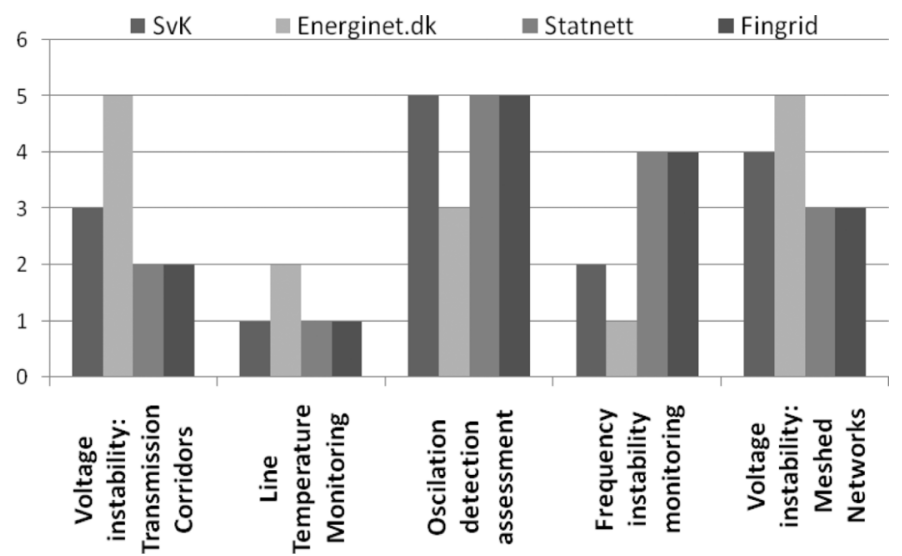

Fig. 2. Applications prioritizations in the Nordic Region.

network is a possible bottleneck in the architecture of these systems since the delays and data quality of the data from PMUs are dependent on the communication infrastructure's capabilities and architecture.

\section{PMU-BASED APPLICATION REQUIREMENTS IN THE NORDIC REGION}

To gather data for building the simulation model and establish a platform for the study, a survey was sent out to TSOs and researchers in the Nordic region involved in PMU projects. The following two sections discuss parts of that survey. A full discussion of the survey can be found in [7]. The purpose of the survey was to gather communication requirements and characteristics of possible monitoring and control applications based on PMUs.

\section{A. Application Prioritization in the Nordic Region}

Five PMU-based applications were presented. The five application functions were: 1) oscillation detection; 2) voltage stability assessment of transmission corridors; 3 ) voltage stability assessment of meshed networks; 4) frequency instability assessment; and 5) line temperature monitoring [8]. The TSOs and researchers were asked to prioritize the importance of each application function in terms of their needs.

Fig. 2 presents the priorities of TSOs in the Nordic region. The figure illustrates the similarities in priorities. There is only a slight variation between the priorities and this can be due to the configuration of the power grid and to the presence of a different generation portfolio. For example, Denmark (Energinet.dk) has a larger percentage of wind power generation than the other nations, which explains why voltage stability applications are more critical to them.

\section{B. WAMC Applications Requirements}

The participants were presented with the same 13 questions for each application function. The purpose of the questions was to collect requirements on such issues as time to complete an assessment, time window for response to phenomena, PMU sample rate requirements and protocols, as well as possible control functions that could be based on these applications. 
TABLE I

OSCILLATION DETECTION REQUIREMENTS FROM INTERVIEWEES

\begin{tabular}{|c|c|c|c|c|c|c|}
\hline Interviewees & $\begin{array}{l}\text { Expected } \\
\text { Latency }\end{array}$ & $\begin{array}{l}\text { Expected } \\
\text { Resolution }\end{array}$ & $\begin{array}{l}\text { Expected Time } \\
\text { Window for } \\
\text { Response }\end{array}$ & $\begin{array}{l}\text { Format/ } \\
\text { Protocol }\end{array}$ & $\begin{array}{l}\text { Time Delay for } \\
\text { Current/Tested } \\
\text { Control Schema }\end{array}$ & $\begin{array}{l}\text { Expected Execution } \\
\text { Time for Control } \\
\text { Schema }\end{array}$ \\
\hline TSO 1 & & $10 \mathrm{~Hz}$ & $\begin{array}{c}\text { Less than } 0.3 \\
\text { seconds }\end{array}$ & $\begin{array}{l}\text { IEEE } 1344 \\
\text { /updating to } \\
\text { C } 37.118\end{array}$ & $\begin{array}{c}\text { To be } \\
\text { determined }\end{array}$ & \\
\hline TSO 2 & $\begin{array}{l}\text { Less than } 2 \\
\text { seconds }\end{array}$ & $10 \mathrm{~Hz}$ & $\begin{array}{c}\text { Fractions of } \\
\text { seconds }\end{array}$ & C37.118 & Not applicable & Fractions of seconds \\
\hline \multicolumn{7}{|l|}{ TSO 3} \\
\hline TSO 4 & $\begin{array}{c}0.25-2 \text { or } 3 \\
\text { seconds }\end{array}$ & $\begin{array}{c}10 / 50 \mathrm{~Hz} \text { for } \\
\text { online/offline } \\
\text { applications }\end{array}$ & & C37.118 & 0.25 seconds & 0.25 seconds \\
\hline $\begin{array}{c}\text { Research } \\
\text { Institute } 1\end{array}$ & Seconds & & $\begin{array}{l}\text { Seconds/Minutes } \\
\text { for automatic/ } \\
\text { manual control }\end{array}$ & & & Seconds \\
\hline $\begin{array}{l}\text { Research } \\
\text { Institute } 2\end{array}$ & $\begin{array}{c}\text { Fractions of } \\
\text { seconds }\end{array}$ & Above $10 \mathrm{~Hz}$ & $\begin{array}{l}\text { Less than } 1 / 10 \text { of } \\
\text { the cycle time of } \\
\text { studied oscillation }\end{array}$ & C37.118 & & \\
\hline $\begin{array}{l}\text { Research } \\
\text { Institute } 3\end{array}$ & 1 second & $50 \mathrm{~Hz}$ & $\begin{array}{l}\text { Less than } 0.2 / 5 \\
\text { seconds for } \\
\text { POD/SPS }\end{array}$ & & $\begin{array}{l}\text { Less than } 1 \\
\text { second }\end{array}$ & \\
\hline NASPI & $1-5$ seconds & $10 \mathrm{~Hz}$ & Seconds & $\begin{array}{c}\text { PDC } \\
\text { Stream/ } \\
\text { C37.118 }\end{array}$ & & \\
\hline
\end{tabular}

The answers were then compared with existing application classifications and requirements from the North American Synchro Phasor Initiative (NASPI) [9].

An example of the results collected is depicted in Table I. The column headings are requirements that were collected for each application. The "Expected Latency" requirement is the amount of time it takes for the phasors to be sent from the PMU until they arrive at the application processing this information. "Expected Resolution" is the number of phasor samples required by the application. The participants also were asked about the format of the data (i.e., which protocol would be used for the transmission of information from the PMU to the WAMC system). The third column indicates the time required for the execution of currently implemented control schemes used to remedy anomalies (e.g., voltage instability control schemes). Finally, the last column indicates the time required for the execution of control schemes that would be based on the results of the PMU-based application.

In general, the requirements collected from the TSOs and researchers dealing with PMU-based applications are similar to specifications outlined by NASPI. But there are some differences, for example, in terms of voltage instability monitoring. TSOs in Nordic countries generally have a higher ambition when considering the response time window in contrast to the NASPI classifications. The requirements for frequency instability monitoring from TSOs in the Nordic region are fairly similar to NASPI classifications while their requirement on the response time window was again longer, when compared to the NASPI classifications.

In terms of line temperature monitoring, the TSOs and researchers in the Nordic region have a tighter requirement on response time of the application which is in the order of sec- onds while in comparison to NASPI's recommendation, the response time is approximately $1 \mathrm{~h}$. Another difference is in the required phasor data resolution where in the Nordic region, it is believed that a low data rate (without specifying exactly) is sufficient while the NASPI suggestion is 30 samples/s.

\section{Modeling Dedicated AND SHAREd COMMUNICATION NETWORKS FOR WAMC SYSTEMS}

A simulation model was built in order to study and illustrate the transmission time of phasor measurements and feedback control signals. By analyzing these low-level qualities, a base is established on which PMU-based systems could be analyzed on a higher level. The simulations are promising and show good correlation with similar studies in other systems.

\section{A. Implementing a PMU Communication Model in OPNET}

To obtain a better estimation of the delays and utilization in PMU communication, a model representing this communication was implemented in OPNET Modeler [10], a communication network simulator. This model was based on possible locations of PMUs in Sweden [11]. The model contains different scenarios corresponding to dedicated and shared network paradigms. The dedicated network paradigm is when the network is utilized solely for PMU communication and where each PMU has a dedicated channel in the network [12], [13]. On the other hand, the shared communication paradigm is when the PMU traffic is sent over a TCP/IP network sending other traffic from RTUs, IEDs, or utility voice-over-IP (VOIP) [14].

It is important to note that the communication model that was built does not represent the actual communication networks used by the Swedish TSO, but is a model with equivalent geographic distances and network architecture. Geographical place- 


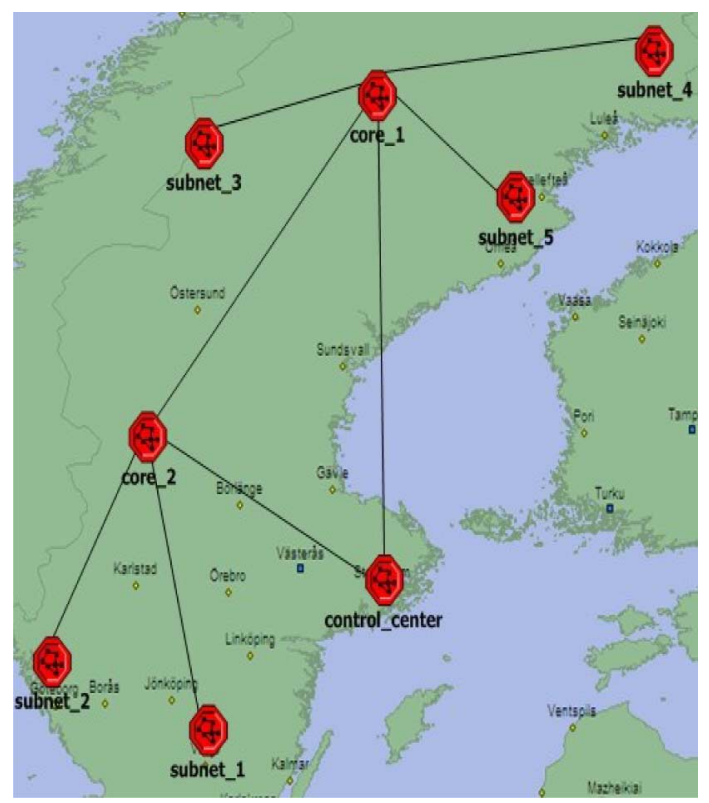

Fig. 3. Fictitious PMU communication network.

ment in the simulation model is important since the model utilizes OPNET's distance-based delay, where the delay due to distance is a component of the total delay between network devices.

\section{B. PMU Communication Network Model}

The PMU communication network model is made up of four scenarios representing different configurations-two support the dedicated and two support the shared communication paradigms.Fig. 3 gives a high-level view of topology and illustrates the geographical scope of the models.

The octagons on the map represent subnets. Subnets are a logical grouping of communication networks. There are two core subnets, core_1 and core_2. The core subnets represent a network of meshed routers (or synchronous digital hierarchy (SDH) switches in the case of the dedicated communications scenario). This was done to represent the complexity and meshed topology that exists in real-world networks.

The traffic generation from PMUs, PDC, and the WAMC devices is similar in all scenarios. The PMUs generates 30 packets/s of size $76 \mathrm{~B}$. Seventy-six bytes are broken down into $16 \mathrm{~B}$ for C37.118 Header and trailer [15], $32 \mathrm{~B}$ for the IP header, and $28 \mathrm{~B}$ for phasor values (e.g., real and imaginary values, etc.) representing one of the possible data loads. The packets from all PMUs are sent to the PDC in the control_center subnet. The PDC receives these packets and generates 30 new packets/s which are sent to the WAMC system. The WAMC system receives these packets and registers delay statistics per packet. Once this is done, the WAMC system model discards the packets. This is because the WAMC system model does not implement any other functionality on incoming packets except for registering delay statistics.

The WAMC system also generates traffic that represents control signals going back to the process. The WAMC traffic is generated in discrete time intervals and sent to a predetermined number of substation switches in various subnets. The difference between the models is in the capacity of the links and the
TABLE II

SUMMARY OF SCENARIOS AND THEIR PARAMETERS IN THE PMU COMMUNICATION NETWORK MODEL

\begin{tabular}{|c|c|c|c|c|}
\hline Scenario & Paradigm & $\begin{array}{c}\text { Bandwidth } \\
\text { Capacity }\end{array}$ & $\begin{array}{c}\text { Samples per } \\
\text { Second }\end{array}$ & $\begin{array}{c}\text { Background } \\
\text { Traffic }\end{array}$ \\
\hline 1 & Dedicated & $64 \mathrm{~Kb} / \mathrm{s}$ & 30 & None \\
\hline 2 & Dedicated & $128 \mathrm{~Kb} / \mathrm{s}$ & 30 & None \\
\hline 3 & Shared & $2 \mathrm{Mb} / \mathrm{s}$ & 30 & $50 \%$ \\
\hline 4 & Shared & $2 \mathrm{Mb} / \mathrm{s}$ & 30 & $70 \%$ \\
\hline
\end{tabular}

protocols utilized. The scenarios varied in the configuration of devices and communication links, a detailed account of the scenarios and parameters discussed in this section, can be found in [16]. Table II provides a summary of the scenarios and the parameters that vary. In all scenarios, the underlining physical media were assumed to be optical fiber. Furthermore, apart from routers and switches that were implemented in the dedicated and shared models as part of the core network and substations, other network devices, such as repeaters, were not modeled.

\section{Simulation Results}

End-to-end (ETE) delay statistics between all PMUs and the PDC were collected. Furthermore, statistics on the transmission time for control signals from the WAMC system to three preselected substation switches was also collected. These preselected substation switches are in substation_3, substation_6 and substation 8 (referring toFig. 3, these are located in subnet_2, subnet_3 and subnet 5, respectively). PMUs in those substations are PMU_3,PMU_6 and PMU_8. The control signals that were sent back to the substations switches were not destined for the PMUs on these substations. The control signals were destined for IEDs or RTUs located on these substations where it is assumed that these devices would execute a control scheme, such as opening a set of breakers.

1) End-to-End Delays From PMUs to PDC: The ETE delay represents the time (in seconds) taken for a packet to reach its destination. In other words, it is the difference between the time a packet arrives at its destination and the time when the packet was created. The delay values were collected separately for each source and destination pair. Each simulated scenario had 13 ETE delay values. Among them, ten were captured from the links from PMUs to PDC and three were captured from the control commands. For the shared communication scenarios, the delays collected included processing delays of intermediary nodes and routing delays due to adaptive routing protocols (specifically, the open shortest path first (OSPF) protocol) in the network. In the dedicated network scenarios, on the other hand, the routing was static and, therefore, delays due to routing were negligible.

ETE delay statistics were collected from dedicated communication scenarios simulation. As mentioned earlier, ten PMUs, in both scenarios, generated a constant traffic of 30 samples/s destined to the PDC. Different channel capacities were used in the simulations based on the existing control network capacity. Table III illustrates the collected ETE delay values from PMUs to PDC for all four scenarios.

The differences between ETE delays within the same scenario were related to geographical distances, and intermediary nodes, 
TABLE III

ETE DELAYS FROM PMUS TO PDC IN ALL FOUR COMMUNICATION SCENARIOS

\begin{tabular}{|c|c|c|c|c|}
\hline $\begin{array}{l}\text { ETE delays } \\
\text { PMUs to } \\
\text { PDC(Sec) }\end{array}$ & $\begin{array}{c}\text { Channel } \\
\text { capacity } \\
64 \mathrm{~Kb}\end{array}$ & $\begin{array}{c}\text { Channel } \\
\text { capacity } \\
128 \mathrm{~Kb}\end{array}$ & $\begin{array}{l}50 \% \text { Back- } \\
\text { ground } \\
\text { traffic }\end{array}$ & $\begin{array}{c}70 \% \text { Back- } \\
\text { ground } \\
\text { traffic }\end{array}$ \\
\hline PMU 1 & 0.041 & 0.021 & 0.016 & 0.028 \\
\hline PMU 2 & 0.045 & 0.024 & 0.016 & 0.028 \\
\hline PMU_3 & 0.037 & 0.02 & 0.012 & 0.021 \\
\hline PMU 4 & 0.031 & 0.016 & 0.012 & 0.021 \\
\hline PMU 5 & 0.065 & 0.035 & 0.019 & 0.033 \\
\hline PMU 6 & 0.072 & 0.039 & 0.019 & 0.033 \\
\hline PMU_7 & 0.042 & 0.042 & 0.018 & 0.031 \\
\hline PMU 8 & 0.046 & 0.025 & 0.017 & 0.029 \\
\hline PMU 9 & 0.039 & 0.02 & 0.013 & 0.023 \\
\hline PMU 10 & 0.015 & 0.008 & 0.005 & 0.011 \\
\hline
\end{tabular}

TABLE IV

ETE DELAYS OF THE CONTROL COMMANDS FOR THE SHARED AND DEDICATED COMMUNICATION SCENARIOS

\begin{tabular}{|c|ccc|}
\hline Scenario & $\begin{array}{c}\text { substation } \\
\text { switch 3 }\end{array}$ & $\begin{array}{c}\text { substation } \\
\text { switch 6 }\end{array}$ & $\begin{array}{c}\text { substation } \\
\text { switch 8 }\end{array}$ \\
\hline $\begin{array}{c}50 \% \text { background } \\
\text { traffic }\end{array}$ & 0.0071 & 0.0107 & 0.0093 \\
\hline $\begin{array}{c}70 \% \text { background } \\
\text { traffic }\end{array}$ & 0.0071 & 0.0107 & 0.0093 \\
\hline $\begin{array}{c}\text { 64Kb dedicated } \\
\text { channel }\end{array}$ & 0.042 & 0.042 & 0.042 \\
\hline $\begin{array}{c}128 \mathrm{~Kb} \text { dedicated } \\
\text { channel }\end{array}$ & 0.021 & 0.022 & 0.021 \\
\hline
\end{tabular}

between PMUs and PDC. Whereas when comparing the ETE delays collected in the $64-\mathrm{kb}$ and the $128-\mathrm{kb}$ scenarios, the delays were reduced, in most cases, approximately by half. In other words, when the channel capacity was doubled, the ETE delay was approximately reduced by half.

As mentioned earlier, in shared communication networks, there would be other traffic on the network coming from other devices (e.g., RTUs) or services (Voice-Over-IP). By adding the constant background traffic to the links in both scenarios, we could analyze the delay variation of PMU traffic between the two scenarios in the presence of different background traffic loads. In a real network, PMUs generate constant traffic in all cases, but the background traffic will actually fluctuate.

2) End-to-End Delays From WAMC toSubstations: The ETE delaysfor the simulated controlsignalsin the dedicatedand shared communication scenarios are presented in Table IV. Three substations switches (substation_switch_3, substation_switch_6, and substation_switch_8, each located in substation_3, substation_6, and substation_8, respectively) were configured to receive commands from the WAMC. The control commands were composed of ten packets each, and were sent from the WAMC at three different times during the simulations.

In the dedicated communication scenarios, the ETE delay of the control signal commands is 42 $\mathrm{ms}, 43 \mathrm{~ms}$, and $43 \mathrm{~ms}$ from the WAMC system to substation_switch_3, substation_switch_6, and substation_
SS LAN

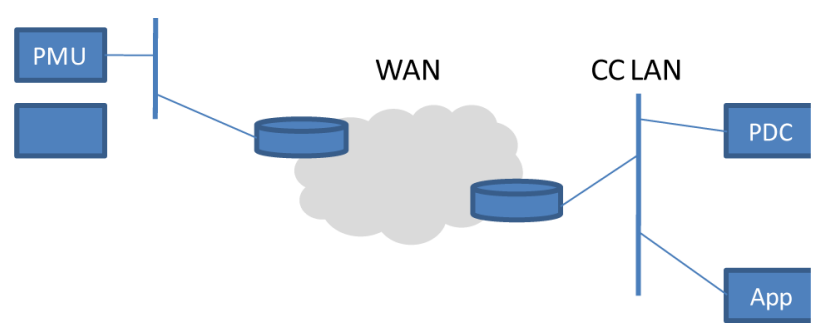

Fig. 4. Utility communication model, showing substation LAN and control center LAN connected by the wide-area network (WAN).

switch_8, respectively, in the case of the dedicated communication with 64-kb/s channels scenario. On the other hand, the ETE delays when the channels capacity is $128 \mathrm{~kb} / \mathrm{s}$ is $21 \mathrm{~ms}, 22 \mathrm{~ms}$, and $21 \mathrm{~ms}$ from the WAMC systems to substation_switch_3, substation_switch_6, and substation_ switch_8, respectively. Again, it can be observed that the delay decreases nearly by half when increasing the channel capacity from 64 to $128 \mathrm{~kb} / \mathrm{s}$.

\section{Time Delay and Data Incompleteness IN WAMC SYSTEMS}

This section discusses the impact of the network delay and PDC parameters on the overall delay and data completeness in WAMC systems. Using the data on the delays collected from the wide-area communication simulation discussed in Section IV, and adding functionality of the PDC for sorting and synchronization, a better understanding of the dynamics of WAMC systems can be studied.

\section{A. Communication Infrastructure}

The communication infrastructure in this part has been simplified as illustrated in Fig. 4. In this abstraction, we assume that the shared communication paradigm is used for PMU communication with the control center. The PMUs are assumed to reside in substations as previously, connected to a local-area network (SS LAN). The SS LAN is, in turn, connected via a substation router to a wide-area network (WAN). The application that utilizes the PMU data resides at a central location connected to a local-area network (CC LAN). Since the application is dependent on data from several PMUs, the data from the PMUs are first passed to the PDC for sorting, after which they are sent to the application.

1) Transmission Delays: In order to specify the transmission delays, we define the following parameters:

$N=\quad$ total number of PMUs;

$i=\quad 1 . . N$;

$T_{i}=\quad$ total end-to-end transmission time for PMU $i$;

$T_{\mathrm{PDC}}=$ processing time in PDC;

$T_{\mathrm{SS}}=$ nominal transmission time on SS LAN; 


$$
\begin{aligned}
T_{\mathrm{CC}}= & \text { nominal transmission time on CC LAN; } \\
T_{\mathrm{WAN}}= & \text { nominal transmission time through WAN } \\
& \text { including routing delays. }
\end{aligned}
$$

With the aforementioned parameters, the total end-to-end transmission time $T_{i}$ for a specific PMU $i$ can be expressed as

$$
T_{i}=T_{\mathrm{SS} i}+T_{\mathrm{WAN} i}+2 T_{\mathrm{CC} i}+T_{\mathrm{PDC} i}
$$

Assuming that $T_{\mathrm{SS} i}=T_{\mathrm{CC} i} \ll T_{\mathrm{WAN} i}$ for all $i$, we chose to represent the transmission time in the WAN $T_{\mathrm{WAN} i}$ as normal distribution with varying mean and standard deviation based as identified in a reference network, and previous simulation studies. The values used for $\mu$ and $\sigma^{2}$ are given in Section IV. Note that in the aforementioned model, delays related to transducers and discrete Fourier transform (DFT) processing of phasor values are not included [17].

With the assumptions from before, we now have

$$
T_{i}=T_{\mathrm{WAN} i}+T_{\mathrm{PDC} i}
$$

The processing time in the PDC depends, in turn, on the implementation of the sorting algorithm in the PDC. Here, a number of variations are possible, and these are discussed in the following section.

2) PDC Model: The most important function of the PDC is to collect or receive the phasor measurements from connected PMUs and to sort them according to their global positioning system (GPS) time stamp. Once a time-stamped set (i.e., all phasors with the same time stamp) is complete, the PDC forwards the phasor set to the applications consuming the data. The PDC can also have other functionalities, such as error checking and archiving, for offline and historical data analysis [6]. In this paper, we consider only the time synchronization functionality. Generally, there are no specified published algorithms and most vendors consider the algorithm as confidential. On the other hand, the main-time synchronization algorithm can be derived from descriptions [18] and the actual requirement of the sorting and synchronization task.

The algorithm used in the simulation described in this paper is as follows. The PDC will group together measurements from the same time stamp into a set. This is done as the measurements arrive to the PDC. Furthermore, the PDC assigns a timeout for each set when the first measurement for each set arrives. The timeout would be the amount of time the buffer is actively waiting for the rest of the phasor measurements with the same time stamp. In some cases, the measurements may be delayed so there will be more than one time-stamped buffer. When the set is full or the set timeout has expired, the PDC will forward the set in the buffer to the applications consuming it.

The timeout per set introduces a ceiling in terms of the delay that can be experienced. If the PMU communication network were to experience abnormal delays or packet loss, then the waiting time parameter ensures that the PDC forwards the phasor measurements in an acceptable time range, without waiting for the delayed measurements to arrive, ensuring that available data, although incomplete, are kept current.

On the other hand, the issue of incomplete data arises where the phasor measurements forwarded to the applications would

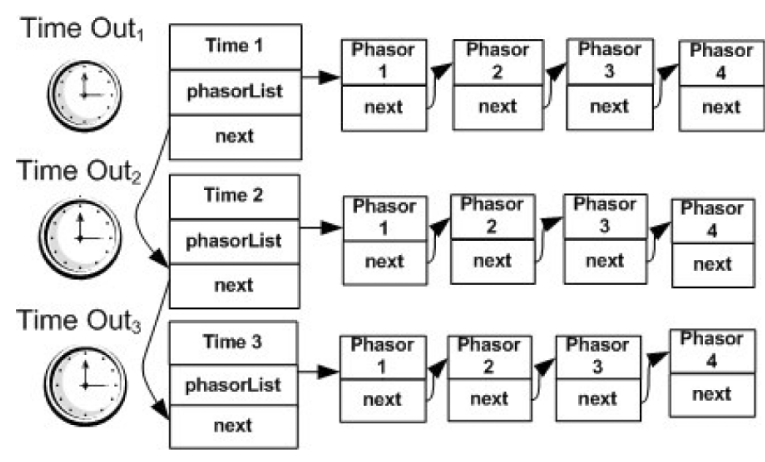

Fig. 5. Time-stamped buffers in the PDC holding measurements in a linked list representation.

be incomplete and miss certain measurements from PMUs on the grid. Fig. 5 illustrates this algorithm.

The amount of time that a packet is delayed in the PDC depends on the following:

- when it arrives (early packets have to wait until all specific timestamps have arrived);

- how many PMUs are sent (more PMUs to wait for);

- how long to wait for the last PMU packet for that time stamp;

- the timeout setting.

This linked-list sorting algorithm can be described as

$$
T_{\mathrm{PDC} i}=f\left(T_{\mathrm{TO}}, T_{\mathrm{WAN} i}\right)
$$

where $\mathrm{T}_{\mathrm{TO}}$ represents the timeout parameter in the PDC sorting algorithm. In the implemented algorithm, the PMU packet with the longest transmission time $\mathrm{T}_{\mathrm{WANi}}$ determines the maximum time that all packets will have to wait

$$
T_{w}=\operatorname{Max}\left(T_{\mathrm{WAN} i}\right) \quad i=1 . . N .
$$

Finally, this gives the complete expression for $T_{\mathrm{PDC} i}$

$$
T_{\mathrm{PDC} i}=T_{\mathrm{sort}}+\operatorname{Min}\left(T_{w} \mid T_{\mathrm{TO}}\right) \text {. }
$$

To study the characteristics of $T_{\mathrm{PDC} i}$ in different system architectures and with different settings for $T_{\mathrm{TO}}$ in networks experiencing different amounts of delay, a simulation study as described in the following section is performed.

\section{B. WAMC Communication Simulations}

To obtain a better estimation of the delays and utilization in PMU communication, a model representing this communication was implemented in OPNET modeler, a communication network simulator.

1) Simulation Model: The overall communication model is made up of submodels that represent PMU devices, the PDC, and the WAMC application server. The PMUs devices are assumed to be in a substation (one PMU per substation)1; furthermore, the PDC and WAMC application server are assumed to be located at the control center. Fig. 6 illustrates the simulation model in a scenario with four PMUs. The number of PMUs varies depending on the scenario set. There are three scenario sets, with four, eight, and sixteen PMUs. 


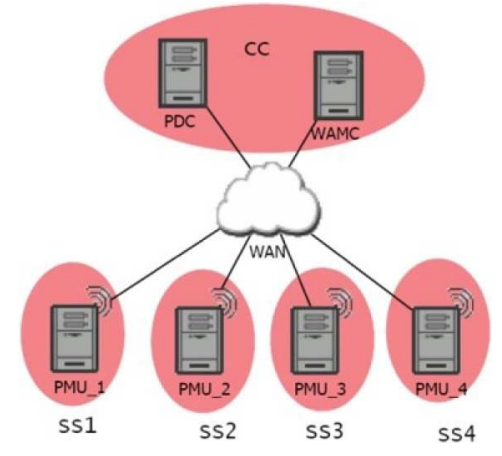

Fig. 6. Topology of scenarios with four PMUs.

TABLE V

Timeout $\left(\mathrm{T}_{\mathrm{TO}}\right.$ ) PARAMETERS UsEd FOR THE PDC MODEL

\begin{tabular}{lllllll}
\hline \multicolumn{7}{c}{$\mathbf{T}_{\mathrm{TO}}$ - time-out parameters (seconds) } \\
\hline 0.015 & 0.025 & 0.03 & 0.035 & 0.045 & 0.05 & 0.055 \\
\hline
\end{tabular}

TABLE VI

Normal DELAY Distribution PARAMETERS

\begin{tabular}{l|ll}
\hline & Mean & Variance \\
\hline Base & 0.0147 & 0.00002 \\
Extended & 0.0454 & 0.000112 \\
\hline
\end{tabular}

2) Simulation Model Scenarios and Parameters: There are a total of 42 scenarios in the simulation model, each with a different combination of values for the parameters. The main parameters that vary in these scenarios are:

- the delay experienced by measurements (from the PMU) traveling to the PDC $\left(\mathrm{T}_{\mathrm{WAN}}\right)$;

- the timeout $\left(\mathrm{T}_{\mathrm{TO}}\right)$ that determines the maximum waiting time at the PDC;

- the number of PMUs in the communication network $(N)$.

The main parameter that is varied in the 42 scenarios is the timeout time. Table V lists the values chosen for the PDC timeout.

The timeout value was used in conjunction with two main delay parameters. The delay parameters were used in the simulation to introduce delay in the transmission of the phasor measurement packets from the PMU to the PDC. The delay parameter is a mean and variance input to a Gaussian probability distribution function which generates random values from the delay. These values are assigned to every packet that is sent. Table VI illustrates the two delay parameters used in the simulation.

The base mean and variance are selected from the previous work aimed at studying transmission delay in communication networks for WAMC systems [16]. This delay parameter is selected to be the standard or base in the simulations. The Extended mean and variance were calculated for usage as values to represent the case of "abnormal" delays that could be experienced in high-speed power system communication networks. The extended delay was applied to a specific number of PMUs and not to all PMUs in any given scenario. This was done to represent the possible delay only from a subset of PMUs that
TABLE VII

Four PMU SCENARIo SET PARAMETER SETTINGS

\begin{tabular}{clcc}
\hline Scenario & Delay Parameter & $\begin{array}{l}\text { Time-Out } \\
\text { Parameter } \\
\left(\mathbf{T}_{\text {TO}}\right)\end{array}$ & $\begin{array}{l}\text { Number of } \\
\text { PMUs with } \\
\text { Extended } \\
\text { Delay }\end{array}$ \\
$\mathbf{1}$ & Base & 0.015 & 0 \\
$\mathbf{2}$ & Base & 0.025 & 0 \\
$\mathbf{3}$ & Base & 0.030 & 0 \\
$\mathbf{4}$ & Base & 0.035 & 0 \\
$\mathbf{5}$ & Base & 0.045 & 0 \\
$\mathbf{6}$ & Base & 0.050 & 0 \\
$\mathbf{7}$ & Base & 0.055 & 0 \\
$\mathbf{8}$ & Base, Extended & 0.015 & 1 \\
$\mathbf{9}$ & Base, Extended & 0.025 & 1 \\
$\mathbf{1 0}$ & Base, Extended & 0.030 & 1 \\
$\mathbf{1 1}$ & Base, Extended & 0.035 & 1 \\
$\mathbf{1 2}$ & Base, Extended & 0.045 & 1 \\
$\mathbf{1 3}$ & Base, Extended & 0.050 & 1 \\
$\mathbf{1 4}$ & Base, Extended & 0.055 & 1 \\
\hline
\end{tabular}

could have been a result from various network or hardware conditions, such as increased traffic on the network segment where the PMUs with extended delay are placed.

Finally, using the combination of delay and timeout parameters, the number of PMUs on the network was varied. For example, Table VII illustrates the scenarios for four PMUs. There are 14 scenarios for 4 PMUs, 7 scenarios experiencing base delay as specified in Table VI, and each scenario has different timeout parameters. The next 7 scenarios also have the same timeout parameter, but in each scenario, 1 PMU will experience an extended delay.

The rest of the scenarios have the same configurations. The number of PMUs with extended delays is increased for the 8 PMUs scenario set to 2 PMUs with an extended delay and in the 16 PMU scenario set to 4 PMUs with extended delay. In short, in the extended delay scenarios, $25 \%$ of the PMUs in the network were configured to experience extended delays.

\section{Analysis}

From the simulations, the mean end-to-end (ETE) delay from the PMUs to the WAMC system and the incompleteness was collected for all of the scenarios. Figs. 7 and 8 illustrate the results for scenarios with 4 and 8 PMUs, respectively.

The incompleteness value in this paper is measured as the percentage of data lost in transit or delayed so that it is not included in the measurement set that is forwarded to the WAMC applications. Incompleteness is therefore the ratio of generated PMU packets that do not reach the WAMC application to the total amount of generated PMU packets.

There are four curves on each graph. For both graphs, two curves illustrate the ETE delay for scenarios with only base delay and extended delay. The other two curves illustrate the incompleteness of the data for the corresponding base and extended delays. In the scenarios with all PMUs experiencing base delays, the incompletes level off at approximately the same time the delay levels off, that is, when the PDC waiting time is 0.045 $\mathrm{s}$. The value for the waiting time is nearly twice the transmission 


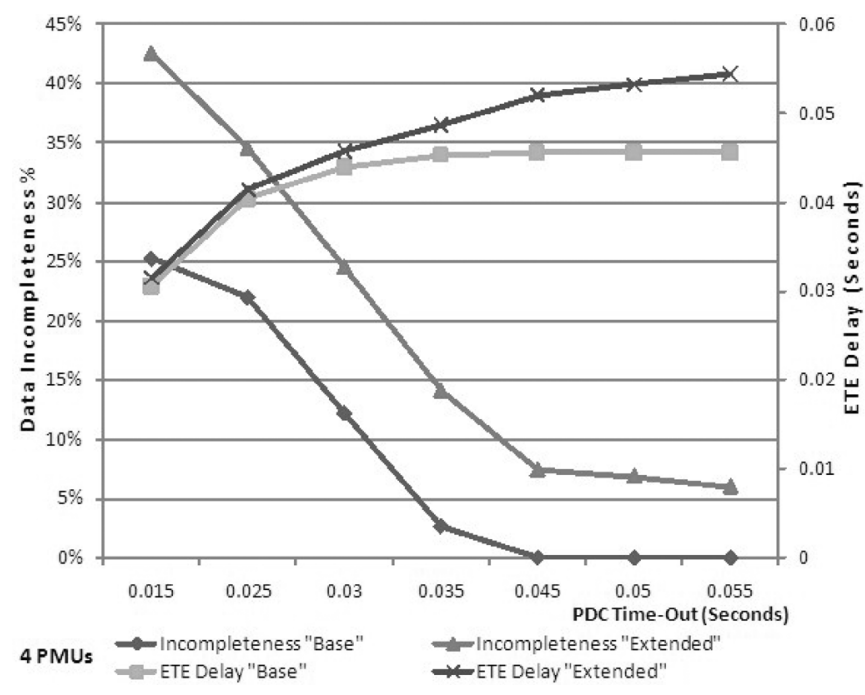

Fig. 7. Four PMUs scenarios.

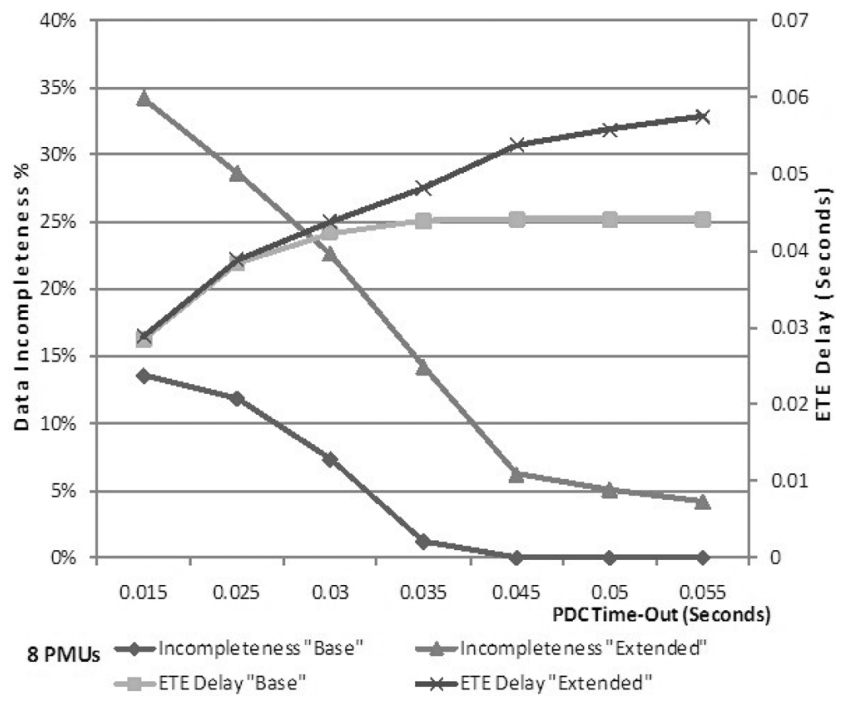

Fig. 8. Eight PMUs scenarios.

time required for the measurements to travel from the PMUs to the PDC.

In the extended delay scenarios, the delay and incompleteness both increase, and in all cases, the incompleteness does not decrease to zero. The data from PMUs with extended delays in all scenarios cause the PDC to wait for the entire waiting time parameter assigned, and then send the data to the WAMC system when the waiting time is up. But as can be observed from the curves, there is significant packet loss resulting in incomplete measurement sets being forwarded to the PDC. While the absolute number of packets lost increases as the number of PMUs is increased, the relative percentage of the packets lost decreases. This is because the proportion of packets experiencing extensive delays decreases as the number of PMUs increases.

In [7], a study on TSO requirements was conducted for widearea applications such as oscillation detection, line temperature monitoring and voltage instability. Specifically, performance requirements and measurement sample rates were collected for these applications. The requirements show that the currency and completeness of the data is an important consideration and the requirements vary depending on the application.

For example, for oscillation damping, the completeness is important but not currency, since the physical changes are not that fast. On the other hand, in transient stability, the timeliness of data is important, but completeness may not be critical, assuming that the critical PMUs located at an important measurement location (a subset of all) are received. Some applications would require both qualities, such as voltage stability, especially in a situation where the phenomenon evolves rapidly.

\section{CONCLUSION AND FURTHER WORK}

WAMC systems intended for centralized applications, such as situational awareness, require data from several separate locations within the power system. The simulations presented in this paper indicate that the geographic distances, background traffic, and architecture of the WAMC system will have an impact on the delay and/or completeness of the PMU data provided to the applications at the central location. Depending on the configuration of the phasor data concentrator (PDC) and the characteristics of the network in terms of delay, some central applications may not receive data of a sufficient quality to provide useful support in transient situations.

The results point out that the communication infrastructure between the PMU and the PDC is not the only bottleneck in the architecture of these systems, and that the PDC settings and performance should also be taken into consideration. The actual use of the PDC model in the study is an important distinction from previous works in the field where the PDC was not taken into account or assumed to be an insignificant part.

Future work will be focused on refinement of these models and possibly on assessing the impact of the delay and data quality on the actual WAMC applications and algorithms. This can be done by using the results provided in this paper and applying other techniques, such as prioritizing important PMUs and applying network quality-of-service (QoS) mechanisms and strategies to these priorities. Another interesting study will be the optimization of incompleteness versus the currency/delay of the data from the PMUs and how this optimization can be built in to the architectures of WAMC systems.

\section{REFERENCES}

[1] A. Phadke, "The wide world of wide-area measurements," IEEE Power Energy Mag., vol. 2, no. 4, pp. 52-65, Sep./Oct. 2008.

[2] D. Karlsson, M. Hemmingsson, and S. Lindahl, "Wide area system monitoring and control," IEEE Power Energy Mag., vol. 2, no. 5, pp. 68-76, Sep./Oct. 2004.

[3] A. Phadke and J. S. Thorp, Synchronized Phasor Measurements and Their Applications. New York: Springer, Jan. 2008.

[4] North American Synchrophasor Initiative. [Online]. Available: www. naspi.org

[5] D. Bakken, C. H. Hauser, H. Gjermundrod, and A. Bose, Towards more flexible and robust data delivery for monitoring and control of the electric power grid. (2007, May 30). School Elect. Eng. Computer Science, Washington State Univ., Tech. Rep. EECS-GS-009. [Online]. Available: http://www.gridstat.net/publications/TR-GS-009.pdf

[6] C. Marinez, M. Parashar, J. Dyer, and J. Coroas, Phasor data requirements for real time wide-area monitoring, control and protection applications. (2005, Jan.). CERTS/EPG, EIPP-Real Time Task Team. [Online]. Available: http://www.naspi.org/resources/archive/rttt/eipp_realtime_group_data_requirements_draft5_jan26_2005.pdf 
[7] M. Chenine, K. Zhu, and L. Nordström, "Survey on priorities and communication requirements on PMU-based applications in the nordic region," presented at the IEEE Power Tech, Bucharest, Romania, Jun. 2009.

[8] M. Zima, M. Larsson, P. Korba, C. Rehtanz, and G. Andersson, "Design aspects for wide-area monitoring and control systems," in Proc. IEEE, May 2005, vol. 93, no. 5, pp. 980-996.

[9] NASPI Data and Network Management Task Team. (2007, Aug. 7)., Phasor application classification. [Online]. Available: http://www.naspi.org/resources/dnmtt/phasorapplicationclassification_20080807.xls

[10] OPNET Inc, OPNET Modeler. [Online]. Available: www.opnet.com

[11] M. Chenine and K. Zhu, PMU-based applications requirements: A survey in the Nordic Region. Royal Inst. Technol., Stockholm, Sweden, Tech. Rep. TRITA-EE 2008:064, 2008.

[12] M. Kim, M. J. Damborg, J. Huang, and S. S. Venkata, "Wide-area adaptive protection using distributed control and high-speed communication," presented at the 14th Power System Computation Conf., Seville, Spain, 2002.

[13] B. Naduvathuparambil, M. C. Valenti, and A. Feliachi, "Communication delays in wide area measurement systems," in Proc. 34th Southeastern Symp. System Theory, 2002, pp. 118-122.

[14] K. H. Mak and B. L. Holland, "Migrating electrical power network SCADA systems to TCP/IP and ethernet networking," Inst. Elect. Eng. Power Eng. J., vol. 16, no. 6, pp. 305-311, Dec. 2002.
[15] IEEE Standard for Synchrophasors for Power Systems, IEEE Std. C37. 118- 2005, 2006. [Online]. Available: ieeexplore.ieee.org

[16] M. Chenine, E. Karam, and L. Nordström, "Modeling and simulation of wide area monitoring and control systems in IP-based networks," presented at the IEEE Power Eng. Soc. Gen. Meeting, Calgary, AB, Canada, Jul. 2009.

[17] J. W. Stahlhut, T. J. Browne, G. T. Heydt, and V. Vittal, "Latency viewed as a stochastic process and its impact on wide area power system control signals," IEEE Trans. Power Syst., vol. 23, no. 1, pp. 84-91, Feb. 2008.

[18] R. Moxley, C. Petras, C. Anderson, and K. Fodero. (2004)., Display and analysis of transcontinental synchrophasors. [Online]. Available: www.selinc.com/literature

Moustafa Chenine (S'09), photograph and biography not available at the time of publication.

Lars Nordstrom (M'05), photograph and biography not available at the time of publication. 